\title{
First detection of Enterocytozoon bieneusi in whooper swans (Cygnus cygnus) in China
}

Yuexin Wang ${ }^{1 \dagger}$, Kaihui Zhang ${ }^{1 \dagger}$, Yifan Zhang ${ }^{2}$, Ke Wang ${ }^{1}$, Azhar Gazizova', Luyang Wang ${ }^{1}$, Letian Cao ,

Yajun Zhang ${ }^{1}$, Jianying Huang ${ }^{1}$, Yuan Cui $^{2}$, Yuxi Zhang ${ }^{3}$ and Longxian Zhang ${ }^{1 *}$ (]

\begin{abstract}
Background: Enterocytozoon bieneusi is a parasite that infects humans and a wide range of other animals. The large migratory waterfowl, the whooper swan (Cygnus cygnus), travels through many cities during its migration and can spread parasites. Despite receiving increasing attention worldwide, there have been no reports of E. bieneusi infection occurring in C. cygnus. Therefore, this study aims to assess the prevalence and genetic characteristics of $E$. bieneusi in $C$. cygnus in Sanmenxia, China.
\end{abstract}

Methods: Altogether, 467 fresh fecal samples were collected in the Swan Wetland Park in Sanmenxia, China. Genomic DNA was extracted from fresh fecal samples $(n=467)$ and E. bieneusi was identified by nested PCR amplification of the internal transcribed spacer (ITS) region. ITS-positive sequences were aligned and phylogenetically analyzed to determine the genotypes of E. bieneusi.

Results: The overall prevalence of E. bieneusi in C. cygnus was 7.49\% (35/467). Sequencing of the 35 positive samples revealed eight known genotypes (EbpA, EbpC, Henan-III, Henan-IV, BEB6, CD9, Peru6 and PtEb IX) and three novel genotypes (CSW1, CSW2 and CSW3). The phylogenetic tree constructed from the ITS sequences showed that seven genotypes (Peru6, EbpA, EbpC, Henan-III, CSW3, Henan-IV and CSW1) clustered within the zoonotic Group 1 while the remaining novel genotype CSW2 clustered within Group 5.

Conclusions: To our knowledge, this is the first report of E. bieneusi in C. cygnus. Of public health significance, our results suggest that migratory $C$. cygnus might play an important role in the water-borne transmission of E. bieneusi. Effective strategies will be necessary to control E. bieneusi infection in C. cygnus, other animals and humans.

Keywords: Enterocytozoon bieneusi, Cygnus cygnus, Zoonotic, Genotype, Migration

\section{Background}

Microsporidia are thought to be zoonotic parasites with the capability of infecting hosts from all major animal taxa worldwide [1]. Of the approximately 1200 species of microsporidia that have been named [2], 17 can infect humans [3]. Up to now, Enterocytozoon bieneusi has the highest worldwide prevalence and is responsible for more

\footnotetext{
*Correspondence: zhanglx8999@henau.edu.cn

${ }^{\dagger}$ Yuexin Wang and Kaihui Zhang equally contributed to this work

${ }^{1}$ College of Animal Science and Veterinary Medicine, Henan Agricultural University, Zhengzhou, Henan, People's Republic of China

Full list of author information is available at the end of the article
}

than $90 \%$ of human microsporidiosis cases [4]. Microsporidia can infect immunodeficient individuals, including those infected with the human immunodeficiency virus and cancer patients. There have been increasing reports of E. bieneusi infections in people with immune disorders, or with sub-optimal immunity such as children and the elderly [5]. There are many ways that E. bieneusi can be contracted, including from food and water. Furthermore, many genotypes that can infect both humans and domestic animals have been identified making microsporidiosis a zoonotic disease [6].

Currently, PCR-based methods are widely used to detect $E$. bieneusi and to analyze its molecular genetic

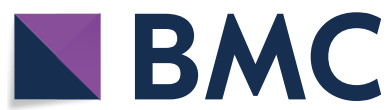

(c) The Author(s) 2020. This article is licensed under a Creative Commons Attribution 4.0 International License, which permits use, sharing, adaptation, distribution and reproduction in any medium or format, as long as you give appropriate credit to the original author(s) and the source, provide a link to the Creative Commons licence, and indicate if changes were made. The images or other third party material in this article are included in the article's Creative Commons licence, unless indicated otherwise in a credit line to the material. If material is not included in the article's Creative Commons licence and your intended use is not permitted by statutory regulation or exceeds the permitted use, you will need to obtain permission directly from the copyright holder. To view a copy of this licence, visit http://creativeco mmons.org/licenses/by/4.0/. The Creative Commons Public Domain Dedication waiver (http://creativecommons.org/publicdomain/ zero/1.0/) applies to the data made available in this article, unless otherwise stated in a credit line to the data. 
characteristics [2]. Nested PCR and sequencing of the internal transcribed spacer (ITS) region of the ribosomal rRNA gene cluster have identified 474 E. bieneusi genotypes globally [6]. Through phylogenetic analysis, $E$. bieneusi genotypes can be divided into 11 groups. Genotypes in Group 1 (e.g. EbpA, EbpC, Peru6, D and type IV, among others) usually infect humans and other animals, with some risk of zoonotic transmission. Some genotypes in Group 2 (e.g. BEB6, BEB4, I and J, among others) were originally classified as ruminant-specific [7] but are believed to have developed reduced host specificity because these genotypes have been identified in other hosts. Significant public health concerns have been raised in connection with the zoonotic potential of these genotypes.

Recently, many E. bieneusi genotypes in Group 1 have been identified worldwide in birds, and these birds may play essential roles in pathogen spread [6]. However, there have been no reports of $E$. bieneusi infection occurring in the whooper swan, Cygnus cygnus. Cygnus cygnus (order Anseriformes, family Anatidae) are large migratory birds with long migratory paths. These birds travel through cities, forests, reservoirs and lakes during migration. To lay the foundations for future studies, the present study employed three fecal sampling sessions timed before the migration of C. cygnus (November and December 2018, and March 2019) to investigate the prevalence of $E$. bieneusi in these birds. We also determined the migration route taken by the winter-migratory
C. cygnus from Sanmenxia, China. If C. cygnus become infected with $E$. bieneusi, they could pose a health threat to other animals including humans, as well as to the environment along their migration pathways. Hence, we carried out this study to assess the prevalence and genetic characteristics of E. bieneusi in C. cygnus in Sanmenxia, China. Our findings will support protection strategies for C. cygnus and public health strategies to address the threat of the zoonotic spread of E. bieneusi.

\section{Methods}

\section{Sample collection}

Altogether, 467 fresh fecal samples were collected during three sampling sessions in Sanmenxia, Henan. The first batch of 237 samples was collected in November 2018, the second batch of 161 samples was collected in December 2018 and the third batch of 69 samples was collected in March 2019. The time of these three sampling periods was in the winter when C. cygnus started wintering or were about to migrate and their numbers were highest. We collected feces from an island in the lake during the feeding period of $C$. cygnus to ensure the samples were fresh (Figs. 1, 2). We then marked and recorded the color and shape of the fecal samples. Approximately 30-50 g of fresh fecal sample (not touching the ground) was collected immediately into a disposable plastic bag after defecation using a sterile disposal latex glove. All fecal samples were stored in $2.5 \%$ potassium dichromate at $4{ }^{\circ} \mathrm{C}$ until processing.

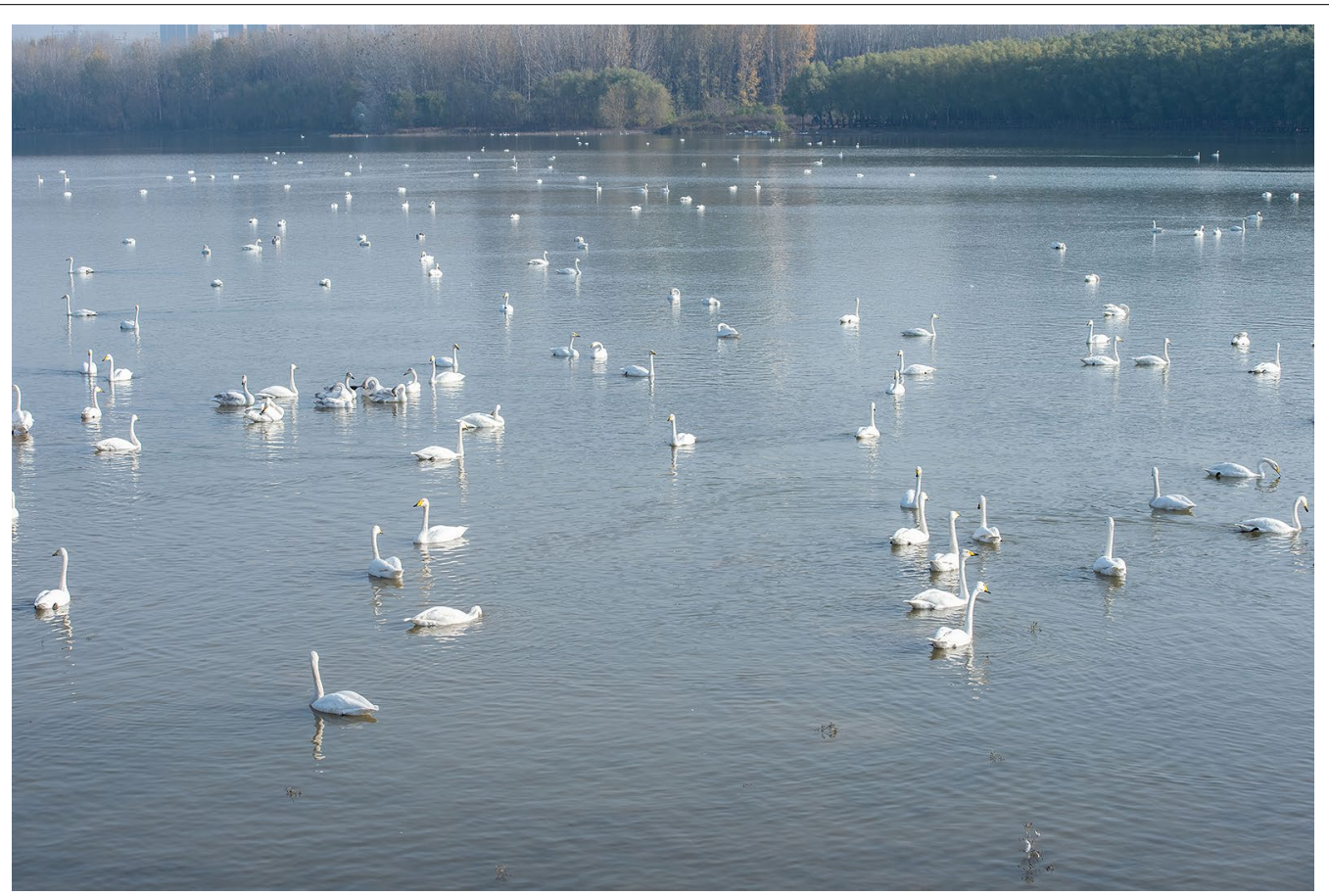

Fig. 1 Whooper swans (Cygnus cygnus) in the Swan Wetland Park in Sanmenxia, China 


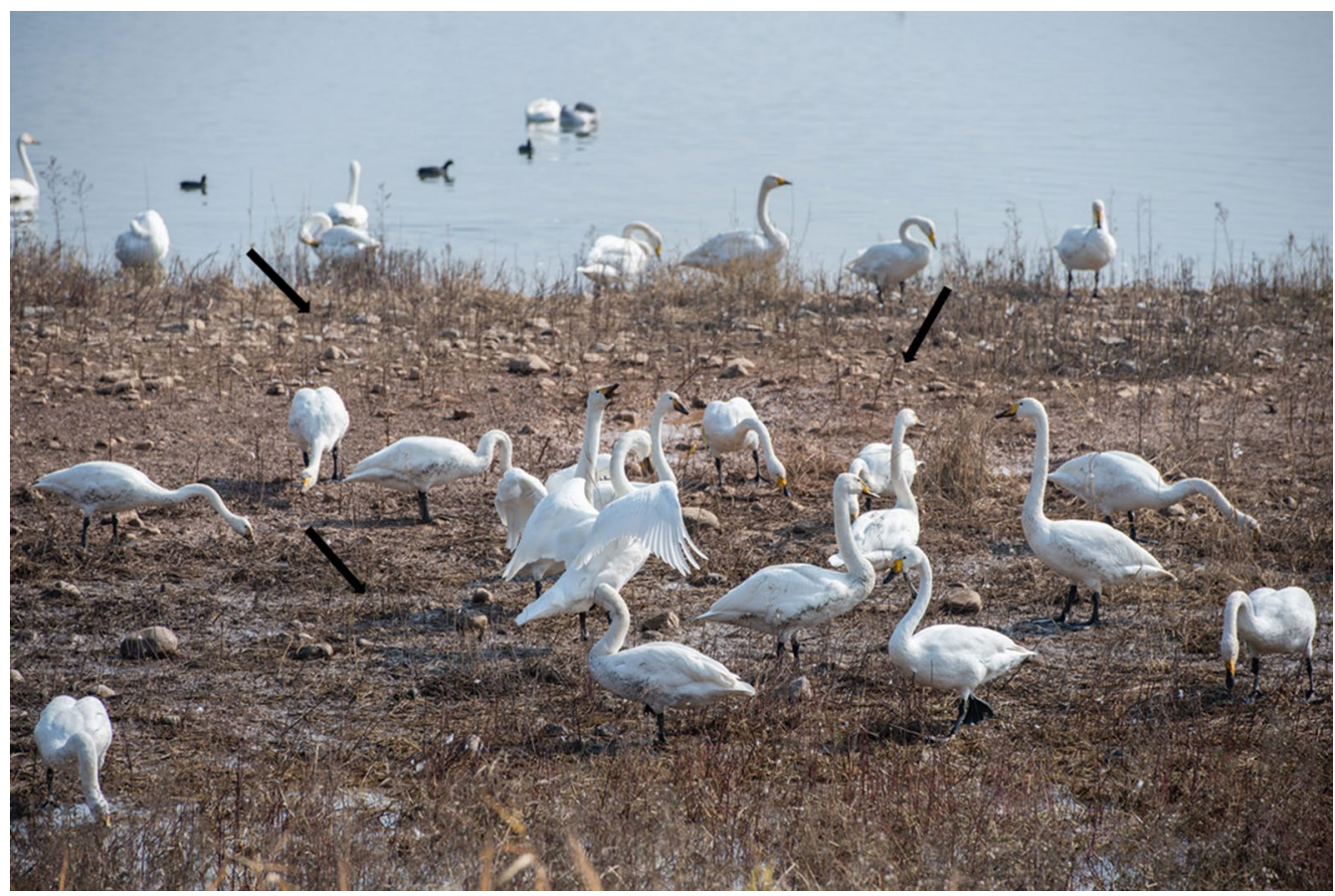

Fig. 2 Sample locations (black arrows) in the Swan Wetland Park in Sanmenxia, China

\section{DNA extraction}

Each fecal sample was washed at least three times with distilled water and centrifuged at $3500 \times g$ for $5 \mathrm{~min}$ to remove potassium dichromate. Before DNA extraction, we transferred up to $200 \mathrm{mg}$ of each stool sample to a $2 \mathrm{ml}$ microcentrifuge tube containing $200 \mathrm{mg}$ of glass beads. The samples were then vortexed at maximum speed until the stool samples were completely homogenized. DNA was extracted using the E.Z.N.A. Stool DNA Kit (Omega Biotek Inc., Norcross, GC, USA) in accordance with the manufacturer's instructions. The extracted DNA was aliquoted and stored at $-20^{\circ} \mathrm{C}$.

\section{PCR amplification and sequence analysis}

Nested PCR and sequencing of the ITS region of E. bieneusi rRNA gene cluster was used to identify genotypes. We used the primer sequences described by Buckholt et al. [8]. The external PCR primers were EBITS3 (5'GGT CAT AGG GAT GAA GAG-3') and EBITS4 (5'TTC GAG TTC TTT CGC GCT C-3') and the internal PCR primers were EBITS1 (5'-GCT CTG AAT ATC TAT GGC T-3') and EBITS2.4 (5'-ATC GCC GAC GGA TCC AAG TG-3'). rTaq DNA polymerase (Takara Bio Inc, Shiga, Japan) was used for PCR amplification. All PCR amplifications included both a positive and negative (no DNA) control and were performed in triplicate. All positive amplification products were confirmed with bidirectional sequencing to ensure accuracy. The sequences were edited using Clustal $\mathrm{X}$, version 2.1 (http://www.clustal.org), Chromas Pro, version 2.1.5.0 (http://technelysium.com.au/ChromasPro.html) and were then aligned with reference sequences downloaded from GenBank. Phylogenetic trees were constructed using the neighbor-joining method (Kimura 2-parameter model) using MEGA, v.7.0 [9], and a bootstrap analysis with 1000 replicates was used to assess tree reliability. The nucleotide sequences generated in this study were deposited in the GenBank database under the accession numbers MN179305-MN179315.

\section{Statistical analysis}

Prevalence differences between different collection times were analyzed using the Chi-square test in SPSS (Release 13.0 standard version; SPSS Inc., Chicago, IL, USA). Values of $P<0.05$ were considered statistically significant. The $95 \%$ confidence intervals (CI) of the prevalence were also calculated by SPSS for Windows.

\section{Results}

\section{Prevalence of E. bieneusi in C. cygnus}

The overall prevalence of E. bieneusi in C. cygnus was 7.49\% (35/467, 95\% CI: 5.10-9.89\%, $\chi^{2}=0.693, d f=2$, $P>0.05)$ across the three sampling sessions (Table 1). There were no significant differences in prevalence across the three sampling sessions [first: $8.44 \%$ (20/137, 95\% 
Table 1 Prevalence and genotypes of E. bieneusi in whooper swans (Cygnus cygnus) in Sanmenxia, China

\begin{tabular}{|c|c|c|c|}
\hline Collection date & $n / N$ & Prevalence (95\% Cl) (\%) & Genotype \\
\hline November 2018 & $20 / 237$ & $8.44(4.87-12.00)$ & $\operatorname{EbpC}(n=7) ; \operatorname{EbpA}(n=7) ;$ Henan-IV $(n=1) ; \operatorname{BEB6}(n=1) ; \operatorname{PtEb} I X(n=2) ; \operatorname{CSW} 1(n=1) ; \operatorname{CSW} 2(n=1)$ \\
\hline December 2018 & $11 / 161$ & $6.83(2.89-10.77)$ & Peru6 $(n=3) ; \operatorname{EbpC}(n=2) ; \operatorname{BEB6}(n=1) ; \operatorname{CD} 9(n=1)$; Henan-III $(n=1) ; \operatorname{PtEb}$ IX $(n=2) ; \operatorname{CSW3}(n=1)$ \\
\hline March 2019 & $4 / 69$ & $5.80(0.14-11.45)$ & Peru6 $(n=3) ; \operatorname{CD9}(n=1)$ \\
\hline Total & $35 / 467$ & $7.49(5.10-9.89)$ & $\begin{array}{l}\operatorname{EbpC}(n=9) ; \operatorname{EbpA}(n=7) ; \text { Peru6 }(n=6) ; \text { PtEb IX }(n=4) ; \text { Henan-IV }(n=1) ; \text { Henan-III }(n=1) ; \operatorname{BEB6} \\
(n=2) ; \operatorname{CD} 9(n=2) ; \operatorname{CSW} 1(n=1) ; \operatorname{CSW} 2(n=1) ; \operatorname{CSW} 3(n=1)\end{array}$ \\
\hline
\end{tabular}

Abbreviations: $\mathrm{n}$, number positive; $\mathrm{N}$, number examined

CI: 4.87-12.00\%), second: $6.83 \%$ (11/161, 95\% CI: 2.89 10.77\%), third: $5.80 \%$ (4/69, 95\% CI: $0.14-11.45 \%)]$.

\section{Genetic characterization and genotype distribution of $E$. bieneusi in C. cygnus}

We identified $11 \mathrm{E}$. bieneusi ITS genotypes, including eight known genotypes (BEB6, EbpC, EbpA, Peru6, Henan-IV, Henan-III, PtEb IX and CD9) and three novel genotypes (CSW1, CSW2 and CSW3) (Table 1). EbpC was the predominant genotype $(n=9)$ causing $25.7 \%$ of E. bieneusi infections, followed by EbpA (20.0\%) and Peru6 (17.1\%). The genotypes EbpC, PtEb IX and BEB6 were detected in both the first and second sampling sessions, and genotype Peru6 was detected in both the second and third sampling sessions.

\section{Phylogenetic analysis}

According to the phylogenetic tree constructed using the ITS sequences from the known novel genotypes identified in this study as well as reference sequences, the genotypes EbpA, EbpC, Henan-III, Henan-IV, Peru6, CSW1 and CSW3 belonged to Group 1. Genotype BEB6 belonged to Group 2 and genotype CSW2 belonged to Group 5. The remaining genotypes (CD9 and PtEb IX) all clustered within Group 11. In this study, we also identified three novel genotypes (CSW1, CSW2 and CSW3). Compared with Henan-IV (GenBank: JQ029727.1), CSW1 contains two single nucleotide polymorphisms (SNPs) in the ITS region (C23T and G107T). Two SNPs were also identified in the ITS region (G43A and G62A) from genotype CSW3 with genotype Henan-IV (GenBank: KJ728794.1), and CSW2 contains one SNP in the ITS region (A32G) with KB-6 (GenBank: JF681180.1) (Fig. 3).

\section{Discussion}

In this study, we found that the overwintering C. cygnus prefers open, food-rich and shallow waters such as lakes rich in aquatic plants, ponds and slow-flowing rivers, especially in the boreal forest. It has been reported that $E$. bieneusi had a wide-host range that includes humans, domestic animals and wild animals [3]. However, E. bieneusi has not been reported in C. cygnus to date. To the best of our knowledge, our study is the first to show the common occurrence of this pathogen in C. cygnus. We found an overall prevalence of $7.49 \%$ of E. bieneusi in overwintering C. cygnus in Sanmenxia, China. Prior to our study, da Cunha et al. [10] had found E. bieneusi in a swan goose from a Brazilian market. However, there is only one study of waterfowl, similar to swans, that reported the prevalence of E. bieneusi in cranes $(12.5 \%$, $7 / 56)$, ducks $(9.7 \%, 6 / 62)$ and geese $(30.8 \%, 8 / 26)$ in China [11]. The prevalence of E. bieneusi in C. cygnus is likely to be affected by multiple factors, including the health of the host, the environmental conditions, and the specificity of the detection methods used [3]. Therefore, it is difficult to explain the different prevalence values reported in [11]. Hence, more research is needed to investigate the situation of migratory animals and waterfowl infections with $E$. bieneusi.

The nucleotide sequence analysis of the ITS region from the E. bieneusi isolates revealed the presence of 11 genotypes including eight known genotypes (EbpA, EbpC, Henan-III, Henan-IV, Peru6, BEB6, CD9 and PtEb IX) and three novel genotypes (CSW1, CSW2 and CSW3). Interestingly, in the present study, EbpC, a zoonotic genotype, which has been detected worldwide in humans and in domestic and wild animals such as pigs, cattle, deer, sheep, dogs, horses, pandas, mice, foxes, beavers and raccoons [6], had the highest prevalence. The genotype EbpA, which has previously been identified in humans in Nigeria and China $[12,13]$ and is widely reported in porcine worldwide $[8,14-16]$, was also found to be highly prevalent. Moreover, two genotypes (HenanIII, Henan-IV) from the present study have only been found in humans, pigs and non-human primates in China [17-19], whereas Peru6 and BEB6 have been identified in various birds, ducks and geese [11]. This is also the first time that known zoonotic genotypes have been reported in C. cygnus, indicating that this species may play an important role in the transmission of E. bieneusi.

Based on the phylogenetic relationships among E. bieneusi isolates, as inferred from the ITS sequences, two genotypes (CSW1 and CSW3) fell into Group 1 indicating that they may imply a potential zoonotic risk. However, we found no significant differences in prevalence 


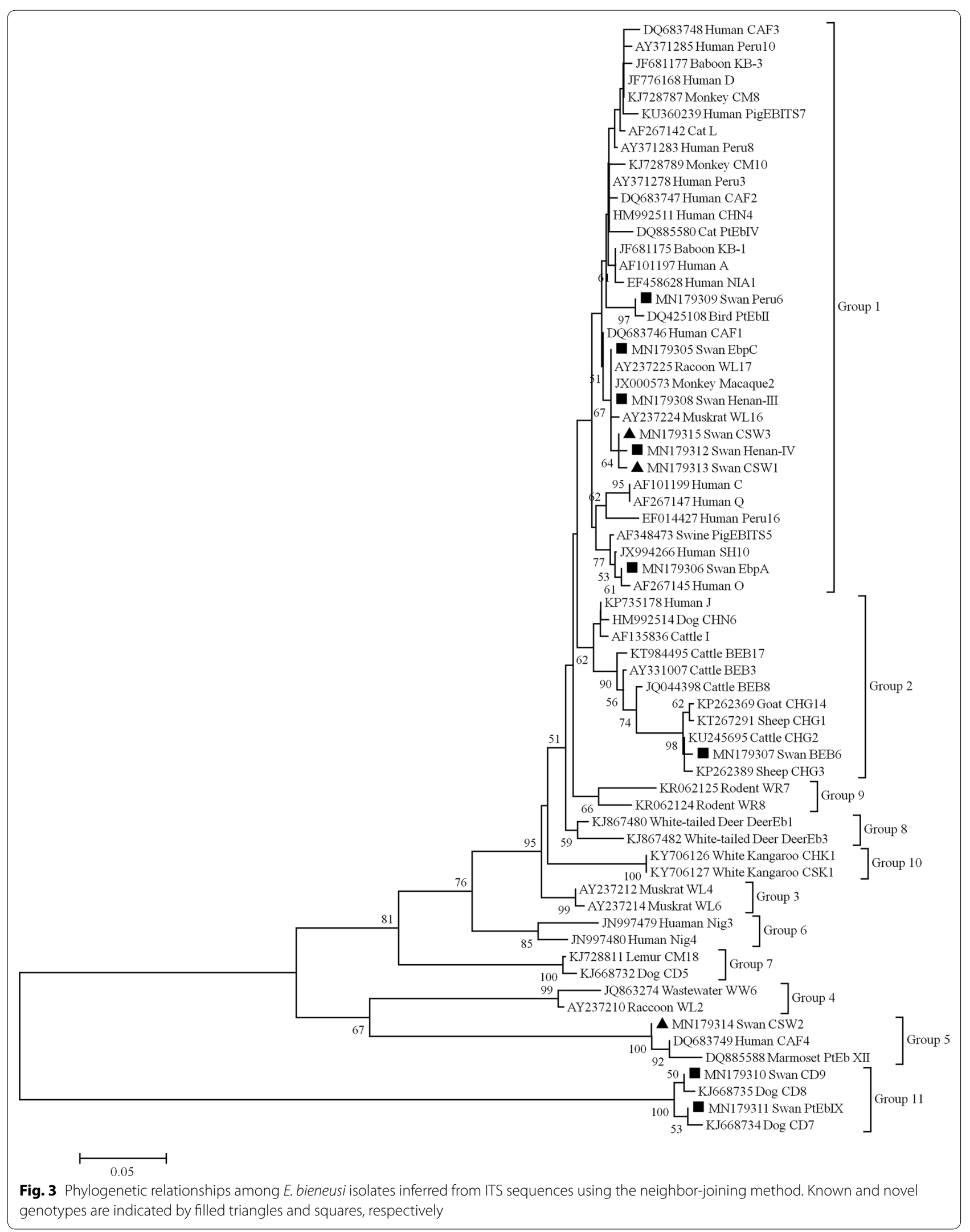




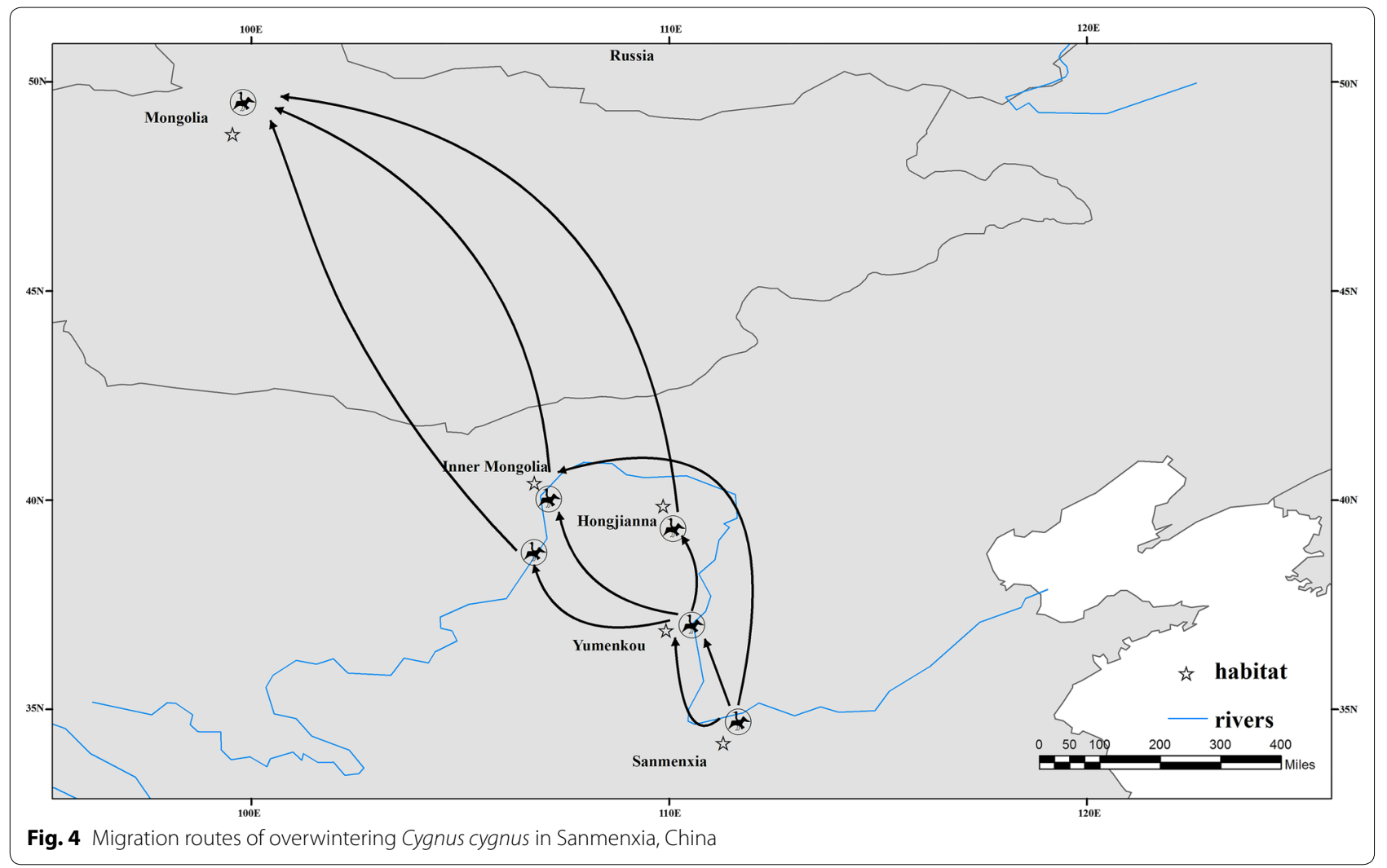

between the three collection time-points. Therefore, we suspect that $C$. cygnus may act as a reservoir host of $E$. bieneusi.

The detection of E. bieneusi spores in multiple water sources (including irrigation water used for crops, domestic water, and effluents from wastewater treatment plants) supports the idea that water may represent a transmission vehicle for this parasite [20-22]. Based on the report from Li [23], we produced a migration map for C. cygnus after they overwintered (Fig. 4), which further illustrates how E. bieneusi might spread. Migration is mainly divided into three stages: the first stage is from Sanmenxia to the Yumenkou wetlands, the second is to the Yellow River in Inner Mongolia, and the third is into central and western Mongolia. Zhang et al. [24] followed overwintering C. cygnus in Sanmenxia using bird bands and GPS technology, and found that at least 40 bodies of water were associated with overwintering $C$. cygnus in Sanmenxia. Thus, E. bieneusi infection in C. cygnus may pose a serious threat to humans and other animals in the region. Although better understanding of E. bieneusi infection in C. cygnus would help with the development of adequate prevention and control strategies against this parasitic infection, further research is needed on tracking the sources of human protozoan pathogens in water bodies. Clearly, regularly surveying harmful microorganisms in water should be a public health priority.

\section{Conclusions}

To the best of our knowledge, the present study is the first to report E. bieneusi in C. cygnus, and to assess the prevalence of this pathogen in overwintering C. cygnus in China. The 11 genotypes identified indicate that the host range for $E$. bieneusi is much wider than previously thought. Importantly, six of the E. bieneusi genotypes from this study have been reported in humans, indicating that $C$. cygnus should be considered a potential source of $E$. bieneusi infections in humans and other animals. Therefore, effective strategies should be designed to control E. bieneusi infection in C. cygnus, other animals, and humans.

\section{Abbreviations}

ITS: internal transcribed spacer; SSU rRNA: small-subunit ribosomal RNA; SNP: single nucleotide polymorphism.

\section{Acknowledgements}

We thank Dr Sandra Cheesman, Liwen Bianji, Edanz Editing China (http://www. liwenbianji.cn/ac), for editing the English text of a draft of this manuscript.

\section{Authors' contributions}

LZ conceived and designed the experiments. YW, KZ and KW performed the experiments and analyzed the data. LW, TC and YZ collected samples. YZ, YC 
and $\mathrm{YY}$ assisted in sampling. YW and LZ wrote the paper. JH took the photographs. All authors read and approved the final manuscript.

\section{Funding}

This study was supported in part by the Natural Science Foundation of Henan Province (162300410129), the National Key Research and Development Programme of China (2017YFD0501305), and the Key Programme of the National Natural Science Foundation of China (31330079).

\section{Availability of data and materials}

Data supporting the conclusions of this article are included within the article Nucleotide sequences were deposited in the GenBank database under the accession numbers MN179305-MN179315.

\section{Ethics approval and consent to participate}

This study adhered strictly to the Regulations for the Implementation of the People's Republic of China on the Protection of Terrestrial Wildlife and the Law of the People's Republic of China on the Protection of Wildlife. The research protocol was reviewed and approved by the Research Ethics Committee of Henan Agricultural University. Samples were collected with the help of the staff at the Sanmenxia Management Office of the Yellow River Wetland National Nature Reserve in Henan Province. No C. cygnus were harmed in this study.

\section{Consent for publication}

Not applicable.

\section{Competing interests}

The authors declare that they have no competing interests.

\section{Author details}

${ }^{1}$ College of Animal Science and Veterinary Medicine, Henan Agricultural University, Zhengzhou, Henan, People's Republic of China. ${ }^{2}$ Sanmenxia Management Office of Henan Yellow River Wetland National Nature Reserve, Sanmenxia, Henan, People's Republic of China. ${ }^{3}$ Sanmenxia Swan Lake National Urban Wetland Park Management Office, Sanmenxia, Henan, People's Republic of China.

Received: 19 September 2019 Accepted: 3 January 2020

Published online: 07 January 2020

\section{References}

1. Stentiford GD, Becnel JJ, Weiss LM, Keeling PJ, Didier ES, Williams BAP, et al. Microsporidia-emergent pathogens in the global food chain. Trends Parasitol. 2016;32:657.

2. Santín M, Fayer R. Microsporidiosis: Enterocytozoon bieneusi in domesticated and wild animals. Res Vet Sci. 2011;90:363-71.

3. Wang S, Wang R, Fan X, Liu T, Zhang L, Zhao G. Prevalence and genotypes of Enterocytozoon bieneusi in China. Acta Trop. 2018;183:142-52.

4. Didier ES, Weiss LM. Microsporidiosis: current status. Curr Opin Infect Dis. 2006;19:485-92.

5. Matos O, Lobo ML, Xiao L. Epidemiology of Enterocytozoon bieneusi infection in humans. J Parasitol Res. 2012;2012:981424.

6. Li W, Feng Y, Santín M. Host specificity of Enterocytozoon bieneusi and public health implications. Trends Parasitol. 2019;35:436-51.

7. Thellier M, Breton J. Enterocytozoon bieneusi in human and animals, focus on laboratory identification and molecular epidemiology. Parasite. 2008;15:349-58

8. Buckholt MA, Lee JH, Tzipori S. Prevalence of Enterocytozoon bieneusi in swine: an 18-month survey at a slaughterhouse in Massachusetts. Appl Environ Micro. 2002;68:2595-9.

9. Kumar S, Stecher G, Tamura K. MEGA7: Molecular evolutionary genetics analysis version 7.0 for bigger datasets. Mol Biol Evol. 2016;33:1870-4.

10. da Cunha MJR, Cury MC, Santín M. Molecular identification of Enterocytozoon bieneusi, Cryptosporidium, and Giardia in Brazilian captive birds. Parasitol Res. 2017;116:487-93.
11. Zhao W, Yu S, Yang Z, Zhang Y, Zhang L, Wang R, Zhang W, Yang F, Liu A. Genotyping of Enterocytozoon bieneusi (Microsporidia) isolated from various birds in China. Infect Genet Evol. 2016;40:151-4.

12. Akinbo FO, Okaka CE, Omoregie R, Dearen T, Xiao L. Molecular characterization of Cryptosporidium spp. in HIV-infected persons in Benin City, Edo State, Nigeria. Fooyin J Health Sci. 2012;2:85-9.

13. Wang L, Xiao L, Duan L, Ye J, Guo Y, Guo M, et al. Concurrent infections of Giardia duodenalis, Enterocytozoon bieneusi, and Clostridium difficile in children during a cryptosporidiosis outbreak in a pediatric hospital in China. PLoS Negl Trop Dis. 2013;7:749-54.

14. Němejc K, Sak B, Květoňová D, Hanzal V, Janiszewski P, Forejtek P, et al. Prevalence and diversity of Encephalitozoon spp. and Enterocytozoon bieneusi in wild boars (Sus scrofa) in central Europe. Parasitol Res. 2014;113:761-7.

15. Li W, Li Y, Li W, Yang J, Song M, Diao R, et al. Genotypes of Enterocytozoon bieneusi in livestock in China: high prevalence and zoonotic potential. PLoS ONE. 2014;9:e97623.

16. Fiuza VR, Oliveira FC, Fayer R, Santín M. First report of Enterocytozoon bieneusi in pigs in Brazil. Parasitol Int. 2015;64:18-23.

17. Wang L, Zhang H, Zhao X, Zhang L, Zhang G, Guo M, et al. Zoonotic Cryptosporidium species and Enterocytozoon bieneusi genotypes in HIV-positive patients on antiretroviral therapy. J Clin Microbiol. 2013;51:557-63.

18. Karim MR, Dong H, LiT, Yu F, Li D, Zhang L, et al. Predomination and new genotypes of Enterocytozoon bieneusi in captive nonhuman primates in zoos in China: high genetic diversity and zoonotic significance. PLOS ONE. 2015;10:e0117991.

19. Li W, Diao R, Yang J, Xiao L, Lu Y, Li Y, et al. High diversity of human-pathogenic Enterocytozoon bieneusi genotypes in swine in northeast China. Parasitol Res. 2014;113:1147-53.

20. Ben Ayed L, Yang W, Widmer G, Cama V, Ortega Y, Xiao L. Survey and genetic characterization of wastewater in Tunisia for Cryptosporidium spp., Giardia duodenalis, Enterocytozoon bieneusi, Cyclospora cayetanensis and Eimeria spp. J Water Health. 2012;10:431-44.

21. Li N, Xiao L, Wang L, Zhao S, Zhao X, Duan L, et al. Molecular surveillance of Cryptosporidium spp., Giardia duodenalis, and Enterocytozoon bieneusi by genotyping and subtyping parasites in wastewater. PLoS Negl Trop Dis. 2012;6:e1809.

22. Graczyk TK, Majewska AC, Schwab KJ. The role of birds in dissemination of human waterborne enteropathogens. Trends Parasitol. 2008;24:55-9.

23. Li S. Home range and migration of wintering whooper swan (Cygnus cygnus) at Sanmenxia reservoir area and spread of HPAI H5N1. Beijing: Chinese Academy of Forestry Sciences; 2017. http://www.caf.ac.cn/. Accessed 20 Aug 2019.

24. Zhang G, Chen L, Li S, Gao R, Ru W, Liu D, et al. The current status of wintering population of whooper swan (Cygnus cygnus) at Sanmenxia reservoir region, China. Chin J Zool. 2016;51:190-7.

\section{Publisher's Note}

Springer Nature remains neutral with regard to jurisdictional claims in published maps and institutional affiliations.

Ready to submit your research? Choose BMC and benefit from:

- fast, convenient online submission

- thorough peer review by experienced researchers in your field

- rapid publication on acceptance

- support for research data, including large and complex data types

- gold Open Access which fosters wider collaboration and increased citations

- maximum visibility for your research: over $100 \mathrm{M}$ website views per year

At BMC, research is always in progress.

Learn more biomedcentral.com/submissions 